\title{
MeV scale leptonic force for cosmic neutrino spectrum and muon anomalous magnetic moment
}

\author{
Joe Sato*t \\ Saitama Univ. \\ E-mail: joe@phy.saitama-u.ac.jp
}

\begin{abstract}
Characteristic patterns of cosmic neutrino spectrum reported by the IceCube Collaboration and long-standing inconsistency between theory and experiment in muon anomalous magnetic moment are simultaneously explained by an extra leptonic force mediated by a gauge field with a mass of the $\mathrm{MeV}$ scale. With different assumptions for redshift distribution of cosmic neutrino sources, diffuse neutrino flux is calculated with the scattering between cosmic neutrino and cosmic neutrino background through the new leptonic force. Our analysis sheds light on a relation among lepton physics at the three different scales, $\mathrm{PeV}, \mathrm{MeV}$, and $\mathrm{eV}$, and provides possible clues to the distribution of sources of cosmic neutrino and also to neutrino mass spectrum. This talk is based on [1].
\end{abstract}

11th International Workshop Dark Side of the Universe 2015

14-18 December 2015

Yukawa Institute for Theoretical Physics, Kyoto University Japan

* Speaker.

${ }^{\dagger}$ A footnote may follow. 


\section{Introduction}

Astrophysics and neutrino physics entered a new era after the discovery of high-energy cosmic neutrino events observed by the IceCube Collaboration [2,3]. The reported spectrum shows some remarkable features, for example, (i) the neutrino flux diminishes steeply as the energy increases, and the best-fit spectral index is $s_{v}=2.5$ [4]; and (ii) there is a gap in the energy range between $400 \mathrm{TeV}$ and $1 \mathrm{PeV}[5,4]$.

In Refs. $[6,7,8,9,10,11,12,13]$, the origin of the gap in the observed spectrum was asked to the attenuation of cosmic neutrino, which is caused by the scattering with cosmic neutrino background $(\mathrm{C} v \mathrm{~B})$ through a new interaction between neutrinos. In such a scenario, the narrow width of the gap can be explained by the resonant behaviour of the scattering. In this study we introduce a new gauged leptonic force $[10,1]$ to explain the gap by calculating diffuse neutrino flux, taking account of the distribution of the source of cosmic neutrino with respect to the redshift. In addition, we show that the leptonic force can simultaneously explain the disagreement between theory and experiment in muon anomalous magnetic moment. Moreover, we search through the model parameter space to find a set of parameters that can reproduce not only the gap but also the sharp edge at the upper end of the cosmic neutrino spectrum. Here we also discuss constraints on the model, such as the neutrino-electron scattering process, invisible decay of a light particle at colliders, big bang nucleosynthesis (BBN), and supernova cooling.

\section{Model and constraints}

We extend the SM of particle physics with a massive vector boson $Z^{\prime}$ that mediates a new leptonic force,

$$
\mathscr{L}_{\text {int }}=g_{Z^{\prime}} Q_{\alpha \beta}\left[\bar{L}_{\alpha} \gamma^{\rho} L_{\beta}+\bar{\ell}_{R} \alpha \gamma^{\rho} \ell_{R_{\beta}}\right] Z_{\rho}^{\prime},
$$

where $L_{\alpha}$ and $\ell_{R \alpha}$ are a lepton doublet and a right-handed charged lepton singlet with flavour $\alpha=$ $\{e, \mu, \tau\}$, respectively. We choose the flavour structure of the interaction as $Q_{\alpha \beta}=\operatorname{diag}(0,1,-1)$, which corresponds to the $U(1)$ gauge interaction associated with muon number minus tau number $\left(L_{\mu}-L_{\tau}\right)[14,15]$. We handle the two parameters, the coupling $g_{Z^{\prime}}$ and the mass $M_{Z^{\prime}}$ of the gauge boson, as parameters that describe the model.

The interaction with neutrinos in Eq. (2.1) is expected to produce the gap and the edge in the cosmic neutrino spectrum through the resonant scattering with $\mathrm{C} v \mathrm{~B}$. In the resonant scattering process $v_{\text {Cosmic }} \bar{v}_{\mathrm{C} v \mathrm{~B}} \rightarrow v \bar{v}$ mediated by $Z^{\prime}$, only cosmic neutrinos having the energy corresponding to the resonance energy $E_{\text {res }}$ are selectively scattered off by $\mathrm{C} v \mathrm{~B}$ on the way from its source to the IceCube $[6,7,8,9,10,12,13]$, which results in the gap around $E_{\text {res }}$. Here, $E_{\text {res }}$ is given as

$$
E_{\mathrm{res}}=\frac{M_{Z^{\prime}}^{2}}{2 m_{v}(1+z)}
$$

where $m_{v}$ stands for a mass of the target $\mathrm{C} v \mathrm{~B}$ and $z$ is the redshift parameter at which the scattering occurs. In Eq. (2.2), the $\mathrm{C} v \mathrm{~B}$ is assumed to be at rest. With an assumption of $m_{v}=\mathscr{O}(0.1) \mathrm{eV}$, the scale of $M_{Z^{\prime}}$ can be estimated as $M_{Z^{\prime}}=\mathscr{O}(1-10) \mathrm{MeV}$ for $E_{\text {res }} \simeq 1 \mathrm{PeV}$. Meanwhile, in order to scatter a sufficient amount of cosmic neutrino during the travel of $\mathscr{O}(1) \mathrm{Gpc}$, the size of the cross 


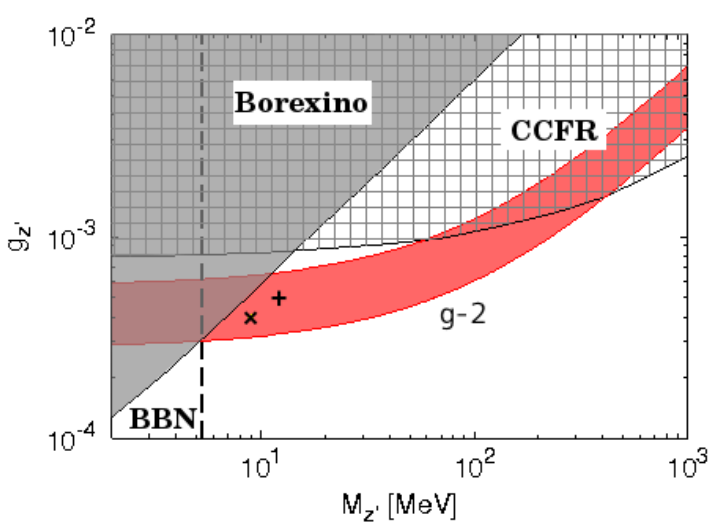

Figure 1: Relevant parameter region and the constraints. The red band represents a parameter region favoured by muon anomalous magnetic moment within $2 \sigma$. The hatched region is excluded by the lepton trident search at the CCFR experiment. The region excluded by the measurement of $v e \rightarrow v e$ at Borexino is filled with a gray colour. The vertical dashed line stands for the lower bound on $M_{Z^{\prime}}$ from BBN. Two symbols + and $\times$ indicate $\left(M_{Z^{\prime}}, g_{Z^{\prime}}\right)=\left(11 \mathrm{MeV}, 5 \times 10^{-4}\right)$ and $\left(9 \mathrm{MeV}, 4 \times 10^{-4}\right)$, respectively, which are used in Sec. IV. See the text for details.

section is required to be larger than $\sim 10^{-30} \mathrm{~cm}^{2}$ at the resonance [6, 7]. In the $L_{\mu}-L_{\tau}$ model, the cross section near the resonance is estimated as

$$
\sigma_{\text {res }}=\frac{2 \pi g_{Z^{\prime}}^{2}}{M_{Z^{\prime}}^{2}} \delta\left(1-\frac{M_{Z^{\prime}}^{2}}{s}\right)
$$

where $s \simeq 2 m_{v} E_{v}$ is the square of the center-of-mass energy in the limit of small $\mathrm{C} v \mathrm{~B}$ momentum. The requirement to the cross section turns out to be $g_{Z^{\prime}} \gtrsim \mathscr{O}\left(10^{-4}\right)$. Putting it all together, the model parameter region that is relevant to the cosmic neutrino spectrum at the energy range around $1 \mathrm{PeV}$ can be deduced as

$$
g_{Z^{\prime}} \gtrsim \mathscr{O}\left(10^{-4}\right) \text { and } M_{Z^{\prime}}=\mathscr{O}(1 \sim 10) \mathrm{MeV} .
$$

The interaction with charged leptons (namely muons) in Eq. (2.1) is responsible for an extra contribution to muon anomalous magnetic moment. The measurement [16] deviates from the SM predictions $[17,18,19,20]$ by around $3 \sigma$. The $Z^{\prime}$ contribution with the combinations of $g_{Z^{\prime}}$ and $M_{Z^{\prime}}$ indicated with the red band in Fig. 1 successfully reproduces the observed value of muon anomalous magnetic moment within $2 \sigma$ errors. In Fig. 1, we also show some experimental constraints (see below) on the model, which pin down the model parameter region onto

$$
g_{Z^{\prime}} \sim \mathscr{O}\left(10^{-4}\right) \text { at } M_{Z^{\prime}}=\mathscr{O}(10) \mathrm{MeV} .
$$

It is worth noting that this parameter region has some overlap with the region Eq. (2.4) required to reproduce the gap and the edge in the cosmic neutrino spectrum.

In the following, we summarize experimental constraints on the $L_{\mu}-L_{\tau}$ model.

$\circ$ Neutrino trident production process: it is a production process of a $\mu^{-} \mu^{+}$pair with a muon neutrino, which results from the scattering of a muon neutrino from a target nucleus observed at several neutrino beam experiments, e.g., the CHARM-II [21] and the CCFR [22]. We adopt the 
95\% C.L. bound of the CCFR experiment, which is shown in Fig. 1 as the hatched region[23].

$\circ$ Neutrino-electron scattering: Although the electron is not charged under the $L_{\mu}-L_{\tau}$ symmetry, $Z^{\prime}$ can interact with an electron through a kinetic mixing $\varepsilon$ between $Z^{\prime}$ and a photon, which is induced by loop diagrams. The total contribution to the kinetic mixing $\varepsilon$ in the $L_{\mu}-L_{\tau}$ model is finite, and it is estimated as

$$
\left|\varepsilon_{\text {loop }}\right|=\frac{8}{3} \frac{e g_{Z^{\prime}}}{(4 \pi)^{2}} \ln \frac{m_{\tau}}{m_{\mu}}=7.2 \cdot 10^{-6}\left(\frac{g_{Z^{\prime}}}{5 \cdot 10^{-4}}\right),
$$

where $e$ is the elementary electric charge. This leads to an extra contribution to the elastic $v e \rightarrow v e$ scattering signal in the solar neutrino measurement at the Borexino experiment $[24,25,26]$. In Fig. 1, the excluded region is filled with a gray colour.

$\circ \mathbf{B B N}$ : A constraint on $M_{Z^{\prime}}$ is derived from BBN. If $Z^{\prime}$ is as light as the temperature at the era of $\mathrm{BBN}$, its existence increases the number of relativistic degrees of freedom, $N_{\mathrm{eff}}$, and the success of the standard BBN might be spoiled, which leads the lower bound $M_{Z^{\prime}} \gtrsim 1 \mathrm{MeV}$ [27]. This condition is always satisfied on the parameter region of our interest. Nevertheless, $Z^{\prime}$ with a mass of $\mathscr{O}(10)$ $\mathrm{MeV}$ may indirectly contribute to $N_{\text {eff }}$ through a raise in the temperature of $v_{\mu}$ and $v_{\tau}$ [12]. In Fig. 1, we display the lower bound on $M_{Z^{\prime}}$ from the indirect contribution with $\Delta N_{\text {eff }}<0.7$ as the vertical dashed line, which is taken from Ref. [12].

\section{Diffuse neutrino flux}

In order to calculate diffuse neutrino flux $\phi_{v_{i}}$ observed at IceCube, we numerically solve the simultaneous partial differential equations with respect to differential number density $\tilde{n}_{v_{i}}\left(E_{v_{i}}, z\right)$ of cosmic neutrino $v_{i}$, which are given in Refs. [28, 7, 13]:

$$
\begin{aligned}
\frac{\partial \tilde{n}_{v_{i}}}{\partial t}= & \frac{\partial}{\partial E_{v_{i}}} b \tilde{n}_{v_{i}}+\mathscr{L}_{v_{i}}-c n_{\mathrm{C} v \mathrm{~B}} \tilde{n}_{v_{i}} \sum_{j} \sigma\left(v_{i} \bar{v}_{j}^{\mathrm{C} v \mathrm{~B}} \rightarrow v \bar{v}\right) \\
& +c n_{\mathrm{C} v \mathrm{~B}} \sum_{j, k} \int_{E_{v_{i}}}^{\infty} \mathrm{d} E_{v_{k}} \tilde{n}_{v_{k}} \frac{\mathrm{d} \sigma\left(v_{k} \bar{v}_{j}^{\mathrm{C} v \mathrm{~B}} \rightarrow v_{i} \bar{v}\right)}{\mathrm{d} E_{v_{i}}}+c n_{\mathrm{C} v \mathrm{~B}} \sum_{j, k} \int_{E_{v_{i}}}^{\infty} \mathrm{d} E_{\bar{v}_{k}} \tilde{n}_{\bar{v}_{k}} \frac{\mathrm{d} \sigma\left(\bar{v}_{k} v_{j}^{\mathrm{C} v \mathrm{~B}} \rightarrow v_{i} \bar{v}\right)}{\mathrm{d} E_{v_{i}}}
\end{aligned}
$$

where $i, j, k=\{1,2,3\}$ are the indices for neutrino mass eigenstates. Similar equation holds for anti-neutrino number density $\tilde{n}_{\bar{v}_{i}}$. The time $t$ is related to redshift $z$ as $\frac{\mathrm{d} z}{\mathrm{~d} t}=-(1+z) H(z)$. Following the discussion in Ref. [29], we treat cosmic neutrino as an incoherent sum of mass eigenstates. The first term on the right-hand side is responsible for energy loss of cosmic neutrino, owing to redshift, and the energy-loss rate $b$ is given with $b=H(z) E_{v}$. The second term represents the influx from sources of cosmic neutrino. In this study, we assume that all sources provide the same spectrum of cosmic neutrino, i.e., $\mathscr{L}_{v_{i}}\left(E_{v_{i}}, z\right)$ is simply parametrized as $\mathscr{L}_{v_{i}}\left(E_{v_{i}}, z\right)=\mathscr{W}(z) \mathscr{L}_{0}\left(E_{v_{i}}\right)$ with the cosmic neutrino spectrum $\mathscr{L}_{0}\left(E_{V}\right)$ from each source and the source distribution $W(z)$ with respect to redshift $z$. Here, the source distribution function is assumed to be common for all the mass eigenstates of cosmic neutrino. We adopt a power-law spectrum, which is characterised by the spectral index $s_{V}$ and the cutoff energy $E_{\text {cut }}$ :

$$
\mathscr{L}_{0}\left(E_{v}\right)=\mathscr{Q}_{0} E_{v}^{-s_{v}} \exp \left[-\frac{E_{v}}{E_{\text {cut }}}\right],
$$


where $\mathscr{Q}_{0}$ is the normalization of the flux, which will be adjusted so as to fit to the observed flux. This type of spectrum typically results from hadronuclear process ( $p p$ inelastic scattering) in the cosmic-ray reservoir, and the values of $s_{V}$ and $E_{\text {cut }}$ are expected to be determined by properties (acceleration rate, i.e., magnetic field and size [30]) of the cosmic neutrino source. The flavour composition of cosmic neutrino from $p p$ reaction is expected to be $\left(v_{e}, v_{\mu}, v_{\tau} ; \bar{v}_{e}, \bar{v}_{\mu}, \bar{v}_{\tau}\right)$ $=(1,2,0 ; 1,2,0)$ at each source, which leads to each mass eigenstate producing approximately with an equal rate. We assume the following function inspired by the star formation rate (SFR) [31] as a test distribution:

$$
\mathscr{W}(z)=\left\{\begin{array}{lc}
(1+z)^{3.4} & 0 \leq z<1 \\
(1+z)^{-0.3} & 1 \leq z \leq 4
\end{array}\right.
$$

The third term represents the outflows caused by the scattering process with $\mathrm{C} v \mathrm{~B}$. Here, the cross section $\sigma$ is

$$
\sigma\left(v_{i} \bar{v}_{j}^{\mathrm{C} v \mathrm{~B}} \rightarrow v \bar{v}\right)=\frac{\left|g_{j i}^{\prime}\right|^{2} g_{Z^{\prime}}^{2}}{6 \pi} \frac{s}{\left(s-M_{Z^{\prime}}^{2}\right)^{2}+M_{Z^{\prime}}^{2} \Gamma_{Z^{\prime}}^{2}},
$$

where $\Gamma_{Z^{\prime}}=g_{Z^{\prime}}^{2} M_{Z^{\prime}} /(12 \pi)$ is the total decay width of $Z^{\prime}$. The coupling $g_{i j}^{\prime}=g_{Z^{\prime}}\left(U^{\dagger}\right)_{i \alpha} Q_{\alpha \beta} U_{\beta j}$ is the coupling in the mass eigenbasis, where $U$ is the lepton mixing matrix. The number density of cosmic neutrino background is given as $n_{\mathrm{C} v \mathrm{~B}}=56(1+z)^{3} / \mathrm{cm}^{3}$ for each degree of freedom. The constant $c$ appearing in the third, forth, and fifth terms is the light speed. The forth and the fifth terms provide the influx from the final states of the scattering process, the so-called regeneration terms. The differential cross sections for cosmic neutrino $v_{i}$ are calculated to be

$$
\frac{\mathrm{d} \sigma\left(v_{k} \bar{v}_{j}^{\mathrm{C} v \mathrm{~B}} \rightarrow v_{i} \bar{v}\right)}{\mathrm{d} E_{v_{i}}}=\frac{\left|g_{j k}^{\prime}\right|^{2} \sum_{l}\left|g_{i l}^{\prime}\right|^{2}}{2 \pi} \frac{m_{v_{j}} E_{v_{i}}^{2}}{E_{v_{k}}^{2}} \times \frac{1}{\left(s-M_{Z^{\prime}}^{2}\right)^{2}+M_{Z^{\prime}}^{2} \Gamma_{Z^{\prime}}^{2}},
$$

We numerically solve these simultaneous partial differential equations Eqs. (3.1) and its antineutrino correspondence After the simultaneous equations are solved, the differential number density $\tilde{n}_{v_{i}}$ of cosmic neutrino at the Earth $(z=0)$ is obtained, and the neutrino flux $\phi_{v_{i}}$ observed at IceCube is calculated as

$$
\phi_{v_{i}}\left(E_{v_{i}}\right)=\frac{c}{4 \pi} \tilde{n}_{v_{i}}\left(E_{v_{i}}, z=0\right) .
$$

\section{Numerical results}

We numerically solve Eqs. (3.1) to calculate the cosmic neutrino flux in the presence of the $L_{\mu}-L_{\tau}$ interaction. In the following calculations, we use the best-fit values of the mixing angles and the mass squared differences from Ref. [32]:

$$
\begin{aligned}
& \sin ^{2} \theta_{13}=0.0234(0.0240), \sin ^{2} \theta_{23}=0.567(0.573), \\
& \sin ^{2} \theta_{12}=0.323, \Delta m_{21}^{2}=7.60 \times 10^{-5}\left[\mathrm{eV}^{2}\right] \\
& \left|\Delta m_{31}^{2}\right|=2.48(2.38) \times 10^{-3}\left[\mathrm{eV}^{2}\right]
\end{aligned}
$$

for the normal (inverted) mass hierarchy, and the $C P$ violating Dirac phase is set to zero. 


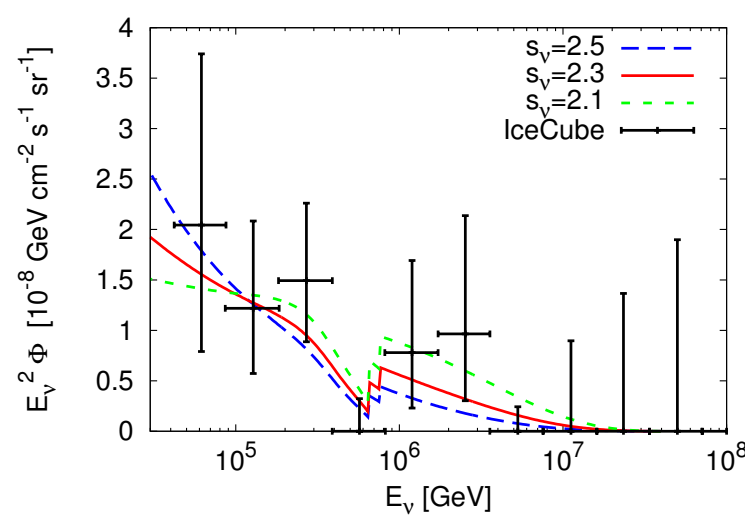

Figure 2: The cosmic neutrino fluxes calculated with the $L_{\mu}-L_{\tau}$ gauge interaction are compared with the three-year IceCube data [5]. The model parameters are taken as $M_{Z^{\prime}}=11 \mathrm{MeV}$ and $g_{Z^{\prime}}=5 \times 10^{-4}$. The lightest neutrino mass is set to be $m_{1}=0.08 \mathrm{eV}$ and the normal mass hierarchy is chosen. The SFR is assumed as the redshift distribution of the cosmic neutrino sources. The cutoff energy of the original flux is placed at $E_{\text {cut }}=10^{7} \mathrm{GeV}$. The three different values of the spectral index $s_{V}$ are examined.

In Fig. 2, we show the cosmic neutrino flux with the attenuation effect by the $L_{\mu}-L_{\tau}$ force and compare the results with three different values of the spectral index $s_{v}$. Here we take the normal hierarchy with the lightest neutrino mass $m_{1}=0.08 \mathrm{eV}[33]$ and set the model parameters as $M_{Z^{\prime}}=11 \mathrm{MeV}$ and $g_{Z^{\prime}}=5 \times 10^{-4}$. For the sources of cosmic neutrinos, we assume the SFR, which is given in Eq. (3.3), as their redshift distribution, and the cutoff energy $E_{\text {cut }}$, which appears in Eq. (3.2), is taken as $E_{\text {cut }}=10^{7} \mathrm{GeV}$. The normalization factor $\mathscr{Q}_{0}$ is adjusted so that the magnitude of the calculated flux fits the observation. As can be seen from the figure, the flux is significantly attenuated around $400 \mathrm{TeV}-1 \mathrm{PeV}$. With a spectrum including the gap, one can expect a relatively good fit to the observation, although the gap will be shallower than the bottom of the calculated spectra once the curves are averaged over each energy bin. The spectrum calculated with the inverted hierarchy is essentially the same as the normal hierarchy

For other studies, such as, the existence of the edge, dependence on source distribution, see the original paper [1].

\section{Discussion and conclusions}

We have introduced an anomaly-free leptonic force mediated by the gauge boson with a mass of the MeV scale in order to simultaneously explain the two phenomena with different energy scales in lepton physics: (i) the disagreement between experimental measurement and theoretical predictions in muon anomalous magnetic moment, and (ii) the characteristic features of the cosmic neutrino spectrum reported by the IceCube Collaboration. Assuming that the PeV cosmic neutrinos are produced after the $p p$ inelastic scattering process in cosmic-ray reservoirs, we have calculated diffuse neutrino flux with the new leptonic force.

We have discussed the relevant constraints, such as the lepton trident process and the observation of a solar neutrino event at the Borexino, and scanned the model parameter space. We 
have found the choices of parameters, which successfully reproduce the measured value of muon anomalous magnetic moment and the gap between $400 \mathrm{TeV}$ and $1 \mathrm{PeV}$ in the IceCube spectrum.

Setting the mass of the leptonic gauge boson to be around $10 \mathrm{MeV}$ and the lightest neutrino mass to be heavier than $1 \times 10^{-3} \mathrm{eV}$, we could arrange the three resonant energies $E_{\text {res }}$ corresponding to three mass eigenstates of cosmic neutrino background to the energy ranges of the gap $\left(E_{V}=400 \mathrm{TeV}-1 \mathrm{PeV}\right)$ and the edge $\left(E_{v} \simeq 3 \mathrm{PeV}\right)$ simultaneously. However, the resonance at the energy corresponding to the edge might be too narrow (and too shallow) to explain the sharp upper end of the spectrum, which is expected from the observation of the IceCube. If one considers the parameter region where the lightest neutrino mass is much lighter than $\mathscr{O}\left(10^{-3}\right) \mathrm{eV}$, momentum distribution of $\mathrm{C} v \mathrm{~B}$ begins to have an impact, and one can expect that the inclusion of the effect would make the resonant region wider.

\section{References}

[1] T. Araki, F. Kaneko, T. Ota, J. Sato and T. Shimomura, MeV scale leptonic force for cosmic neutrino spectrum and muon anomalous magnetic moment, Phys. Rev. D93 (2016) 013014, [1508. 07471 ].

[2] IceCube Collaboration collaboration, M. Aartsen et al., First observation of PeV-energy neutrinos with IceCube, Phys.Rev.Lett. 111 (2013) 021103, [1304 . 5356].

[3] ICECUBE collaboration, M. Aartsen et al., Evidence for High-Energy Extraterrestrial Neutrinos at the IceCube Detector, Science 342 (2013) 1242856, [1311 . 5238].

[4] ICECUBE collaboration, M. G. Aartsen et al., A combined maximum-likelihood analysis of the high-energy astrophysical neutrino flux measured with IceCube, 1507.03991.

[5] IceCube Collaboration collaboration, M. Aartsen et al., Observation of High-Energy Astrophysical Neutrinos in Three Years of IceCube Data, Phys.Rev.Lett. 113 (2014) 101101, [1405.5303].

[6] K. Ioka and K. Murase, IceCube PeV-EeV neutrinos and secret interactions of neutrinos, PTEP 2014 (2014) 061E01, [1 404.2279$].$

[7] K. C. Y. Ng and J. F. Beacom, Cosmic neutrino cascades from secret neutrino interactions, Phys.Rev. D90 (2014) 065035, [1 404 .2288].

[8] M. Ibe and K. Kaneta, Cv B absorption line in the neutrino spectrum at IceCube, Phys.Rev. D90 (2014) 053011, [1407.2848].

[9] K. Blum, A. Hook and K. Murase, High energy neutrino telescopes as a probe of the neutrino mass mechanism, 1408.3799 .

[10] T. Araki, F. Kaneko, Y. Konishi, T. Ota, J. Sato et al., Cosmic neutrino spectrum and the muon anomalous magnetic moment in the gauged $L_{\mu}-L_{\tau}$ model, Phys.Rev. D91 (2015) 037301, [1409.4180].

[11] J. F. Cherry, A. Friedland and I. M. Shoemaker, Neutrino Portal Dark Matter: From Dwarf Galaxies to IceCube, 1411.1071.

[12] A. Kamada and H.-B. Yu, Coherent Propagation of PeV Neutrinos and the Dip in the Neutrino Spectrum at IceCube, 1504.00711.

[13] A. DiFranzo and D. Hooper, Searching for MeV-Scale Gauge Bosons with IceCube, 1507 . 03015. 
[14] R. Foot, New Physics From Electric Charge Quantization?, Mod.Phys.Lett. A6 (1991) 527-530.

[15] X. He, G. C. Joshi, H. Lew and R. Volkas, NEW Z-prime PHENOMENOLOGY, Phys.Rev. D43 (1991) $22-24$.

[16] MuOn G-2 collaboration, G. Bennett et al., Final Report of the Muon E821 Anomalous Magnetic Moment Measurement at BNL, Phys.Rev. D73 (2006) 072003, [hep-ex/ 0602035 ].

[17] M. Davier, A. Hoecker, B. Malaescu and Z. Zhang, Reevaluation of the Hadronic Contributions to the Muon g-2 and to alpha(MZ), Eur. Phys. J. C71 (2011) 1515, [1010.4180].

[18] K. Hagiwara, R. Liao, A. D. Martin, D. Nomura and T. Teubner, $(g-2)_{\mu}$ and alpha $\left(M_{Z}^{2}\right)$ re-evaluated using new precise data, J.Phys. G38 (2011) 085003, [1105.3149].

[19] T. Aoyama, M. Hayakawa, T. Kinoshita and M. Nio, Complete Tenth-Order QED Contribution to the Muon g-2, Phys. Rev. Lett. 109 (2012) 111808, [1205.5370].

[20] A. Kurz, T. Liu, P. Marquard and M. Steinhauser, Hadronic contribution to the muon anomalous magnetic moment to next-to-next-to-leading order, Phys. Lett. B734 (2014) 144-147, [1403. 6400 ].

[21] CHARM-II collaboration, D. Geiregat et al., First observation of neutrino trident production, Phys. Lett. B245 (1990) 271-275.

[22] CCFR Collaboration collaboration, S. Mishra et al., Neutrino tridents and W Z interference, Phys.Rev.Lett. 66 (1991) 3117-3120.

[23] W. Altmannshofer, S. Gori, M. Pospelov and I. Yavin, Neutrino Trident Production: A Powerful Probe of New Physics with Neutrino Beams, Phys.Rev.Lett. 113 (2014) 091801, [1 406 . 2332].

[24] R. Harnik, J. Kopp and P. A. Machado, Exploring nu Signals in Dark Matter Detectors, JCAP 1207 (2012) 026, [1202.6073].

[25] S. K. Agarwalla, F. Lombardi and T. Takeuchi, Constraining Non-Standard Interactions of the Neutrino with Borexino, JHEP 12 (2012) 079, [1207.3492].

[26] S. Bilmis, I. Turan, T. M. Aliev, M. Deniz, L. Singh and H. T. Wong, Constraints on Dark Photon from Neutrino-Electron Scattering Experiments, 1502.07763.

[27] B. Ahlgren, T. Ohlsson and S. Zhou, Comment on âĂIJIs Dark Matter with Long-Range Interactions a Solution to All Small-Scale Problems of ÎŻ Cold Dark Matter Cosmology?âĂİ, Phys. Rev. Lett. 111 (2013) 199001, [1309.0991].

[28] Y. Farzan and S. Palomares-Ruiz, Dips in the Diffuse Supernova Neutrino Background, JCAP 1406 (2014) 014, [1401.7019].

[29] Y. Farzan and A. Y. Smirnov, Coherence and oscillations of cosmic neutrinos, Nucl.Phys. $\mathbf{B 8 0 5}$ (2008) 356-376, [0803.0495].

[30] A. M. Hillas, The Origin of Ultrahigh-Energy Cosmic Rays, Ann. Rev. Astron. Astrophys. 22 (1984) 425-444.

[31] H. Yuksel, M. D. Kistler, J. F. . Beacom and A. M. Hopkins, Revealing the High-Redshift Star Formation Rate with Gamma-Ray Bursts, Astrophys.J. 683 (2008) L5-L8, [0 804.4008 ].

[32] D. Forero, M. Tortola and J. Valle, Neutrino oscillations refitted, 1405.7540.

[33] Planck Collaboration collaboration, P. Ade et al., Planck 2013 results. XVI. Cosmological parameters, Astron.Astrophys. (2014) , [1303.5076]. 